\title{
LA INFANCIA MÁS VULNERABLE, UN COMPROMISO IMPOSTERGABLE
}

\author{
Autora: Soledad Cardozo Salazar \\ scardozo@aldeasinfantiles.es \\ Responsable de Relaciones con Empresas y Fundaciones, \\ Aldeas Infantiles SOS España
}

\section{Resumen}

Los ODS han venido para quedarse y las ONG somos un socio clave y un puente necesario entre todos los sectores implicados.

Desde Aldeas Infantiles SOS participamos en la elaboración de la Agenda 2030, logrando, junto con otras organizaciones dedicadas a infancia, que los niños que han perdido el cuidado de sus padres o que están en riesgo de perderlo sean tenidos en cuenta.

La Agenda 2030 representa una oportunidad para trabajar en red, que para las ONG supone un nuevo desafío. En Aldeas Infantiles SOS hemos alineado el trabajo que desempeñamos en nuestros programas con este desafío, trabajando junto con los diferentes sectores y creando alianzas para poder conseguir un mundo más justo y llegar a muchos más niños. 
Desde Aldeas Infantiles SOS contribuimos directamente a la consecución de 5 objetivos, pero incidimos también en otros, por la interconexión existente entre los distintos ODS.

Además, medimos el resultado que ha tenido y tendrá nuestro trabajo en relación al cumplimiento de los ODS: dado nuestro impacto en las vidas de los exparticipantes, es razonable esperar que los resultados positivos que vemos en ellos igualmente deban verse reflejados en las vidas de los participantes actuales y futuros hasta 2030.

A modo ejemplificativo, desde nuestros programas, apoyamos a los niños y jóvenes para que tengan acceso a una educación de calidad y reciban formación en habilidades. Pues bien, el $82 \%$ de los exparticipantes han tenido resultados positivos en estas áreas, por lo que podemos esperar también que el $82 \%$ de los participantes actuales y futuros sean positivamente impactados en términos de educación y habilidades, lo que supone 5,3 millones de niños hasta 2030.

Es evidente, por ello, que invertir en la infancia temprana trae consigo resultados positivos y rentables para las empresas y para la sociedad en su conjunto, pues lo que le suceda en esta etapa de la vida a un niño, determinará su futuro y, por tanto, la base sobre la que se construyen las sociedades.

Palabras Clave: infancia vulnerable; cuidado parental; ODS; Agenda 2030; impacto; Aldeas Infantiles; desarrollo sostenible; responsabilidad social.

\title{
The most vulnerable childhood, an impossterable commitment
}

\begin{abstract}
SDGs have come to stay and NGOs are a key partner and a necessary bridge between all sectors involved.

SOS Children's Villages has been involved in working on the 2030 Agenda, achieving, together with other organizations for children, that children who have lost parental care or are at risk of losing it were taken into account.

2030 Agenda is an opportunity for networking, which is a new challenge for NGOs. In SOS Children's Villages Spain we have aligned the work we do in our programs with this challenge, working together with different sectors and creating partnerships to build a fairer world and reach many more children.

From SOS Children's Villages Spain, we contribute directly to the achievement of 5 objectives, but we also have an impact on others, because of the interconnection between the different SDGs.

In addition, we measure the result that our work has had and will have in relation to the fulfillment of the SDGs: given our impact on the exparticipants'
\end{abstract}


lives, it is reasonable to expect that the positive results seen in them should also be reflected in the current and future participants' lives until 2030.

As an example, from our programs, we support children and young people so that they have access to quality education and receive training in skills. $82 \%$ of exparticipants have had positive results in these areas, so we can also expect that $82 \%$ of current and future participants will be positively impacted in terms of education and skills, which means 5.3 million children by 2030 .

It is clear, therefore, that investing in early childhood brings positive and profitable results for business and society as a whole, because what happens to a child at this stage of life will determine his or her future and, therefore, the basis on which societies are built.

Keywords: vulnerable childhood; parental care; SDG; 2030 Agenda; impact; SOS Children's Villages; sustainable development; social responsibility.

\section{INTRODUCCIÓN}

Los Objetivos de Desarrollo Sostenible han trazado un camino para todos los agentes sociales, incluido el Tercer Sector. Las ONG llevamos años contribuyendo con muchos de ellos, pero con esta nueva Agenda se nos marca un camino único para todos, con unos objetivos claros y compartidos.

La Agenda también nos legitima de una vez por todas como agente indispensable y decisivo a la hora de tomar decisiones conjuntas en torno a temas globales, como el fin de la pobreza, la desigualdad, el hambre o la injusticia. Las ONG somos un socio clave y esencial, somos un intermediario activo y un puente necesario entre la ciudadanía, los poderes públicos y el sector empresarial.

La Agenda 2030 también ha significado una nueva oportunidad para favorecer el trabajo en red y la creación de alianzas entre organizaciones y entre sectores, algo a lo que no estábamos tan acostumbradas las ONG. Estamos, por tanto, llamadas a crear redes que permitan el intercambio de experiencias y conocimientos y la búsqueda de soluciones conjuntas, con mayor impacto y pertinencia. 


\section{EL COMPROMISO DE ALDEAS INFANTILES SOS HACIA UN MUNDO MÁS JUSTO E IGUALITARIO}

Desde Aldeas Infantiles SOS consideramos crucial impulsar el cumplimiento de los ODS, no solo en España sino en el mundo. Por ello, frente a la aparición de la Agenda 2030 en el 2015, desde nuestra acción social hemos analizado en detalle a qué ODS contribuimos de manera directa con nuestro trabajo.

De este modo, en ese año iniciamos un proceso de reflexión y análisis en los 136 países y territorios en los que formamos parte de la Federación, que culminó con un plan estratégico a 2030, donde trazamos el camino que debemos recorrer, con una firme pisada, para contribuir a la consecución de los ODS más vinculados con nuestra Misión y Visión.

Al prevenir la separación de padres e hijos en situación de vulnerabilidad, garantizar un cuidado alternativo de calidad para los niños que perdieron el cuidado parental y preparar a los jóvenes para una vida independiente, desde Aldeas Infantiles SOS contribuimos fundamentalmente a la consecución de 5 objetivos: ODS 1 (fin de la pobreza), ODS 4 (educación de calidad), ODS 8 (trabajo decente y crecimiento económico), ODS 10 (reducción de las desigualdades) y ODS 16 (paz, justicia e instituciones sólidas). Asimismo, nuestra labor también influye positivamente en el logro de aquellas metas vinculadas a la salud y el bienestar, la igualdad de género y la creación de alianzas multi-actor.

Por otra parte, es interesante señalar la interconexión existente entre los distintos ODS y cómo desde lo local también estamos influyendo a lo global. Así, si ofrezco apoyo a un joven en situación de vulnerabilidad para que pueda realizar sus estudios, no solo estoy influyendo en el Objetivo 4 de garantizar una educación de calidad, sino también en el Objetivo 10, que habla de la reducción de las desigualdades.

A su vez, participamos de manera activa en el proceso de discusión y elaboración de la Agenda 2030, mediante nuestra representante permanente en Naciones Unidas. Junto a otras organizaciones dedicadas a la infancia, logramos que los niños que han perdido el cuidado de sus padres o que están en riesgo de perderlo sean tomados en cuenta en esta agenda. Así, se incorpora el principio Leave no one behind (no dejar a nadie atrás), que expresa la voluntad de que ningún objetivo puede cumplirse a menos que se cumpla para todos.

No obstante, hace más de 50 años que Aldeas Infantiles SOS está trabajando activamente en los ODS en España. Nuestros orígenes y quehacer están enfocados en atender a las personas más vulnerables y muchas veces invisibles, en dejar nadie atrás. La trayectoria y alcance de nuestra intervención social dirigida a la infancia y sus familias, nos permite hacer frente con firmeza y resolución, con experiencia y aprendizaje, los retos que la Agenda 2030 plantea. Podemos decir que la Agenda 2030 es también nuestra Agenda. 
En España hemos asumido e integrado plenamente estas 5 metas en nuestros planes de trabajo para dar respuestas adecuadas a los niños que están en riesgo de perder el cuidado parental, a los que ya los han perdido y acompañando a los jóvenes más vulnerables hacia su plena integración social. Colaboramos especialmente con la Administración, las empresas y con entidades sociales relacionadas con el bienestar de la infancia. Sabemos que el objetivo es de todos y establecer redes basadas en el respeto y compromiso con todos los actores involucrados es la única manera de conseguirlo.

Nuestro proyecto educativo de intervención directa también contempla de manera transversal que los niños, los jóvenes y las familias conozcan, reflexionen y se comprometan con la consecución de esos objetivos, partiendo de sus propias realidades: fomentando el valor de la paz y la justicia en sus hogares, cuidando y respetando el entorno natural donde se desenvuelven, reciclando y reutilizando, entre otras.

Además de nuestro trabajo en atención directa a la infancia, nos hemos comprometido con la sensibilización de los ODS, en distintos ámbitos de influencia:

- con los grupos sociales externos con los que interactuamos, como donantes privados, empresas y administraciones locales, con quienes buscamos instancias de intercambio y aprendizaje;

- a través de nuestra estrategia de incidencia política, basada en la colaboración y seguimiento del cumplimiento de los planes presentados por el gobierno en Naciones Unidas, especialmente con aquellos ODS relacionados con nuestra Misión;

- a través de nuestro programa "Educación en Valores", dirigido a alumnos de Primaria (Abraza tus valores) y Secundaria (Párate a pensar) que venimos ofreciendo a los centros educativos desde 1998 y en los que participan más de 300.000 niños y adolescentes por año. Desde 2019 este programa tiene como objetivo la difusión de los ODS y la reflexión de los alumnos y de sus familias en torno a valores relacionados con estas metas.

- Estamos convencidos que, en la medida en que el compromiso con un mundo más justo e igualitario y respetuoso con el medioambiente sea colectivo y compartido, permeando todas las esferas de la sociedad, solo así seremos capaces de responder a tiempo a las metas globales. Y para ello, brindar información a las personas y grupos, haciéndolas visualizar la importancia de su accionar para un mundo mejor y sostenible, resulta clave. 


\section{NIÑOS SIN CUIDADO PARENTAL Y LA AGENDA 2030}

La familia es el lugar de referencia por excelencia para todas las personas. Es donde cada individuo debe sentirse querido, cuidado y protegido. Para los niños, niñas y adolescentes la familia es el núcleo central de su protección. Crecer en familia trae consigo consecuencias positivas en su desarrollo integral e impacta directamente en sus logros y oportunidades de futuro. Vivir en familia es reconocido como un derecho humano y, como tal, debe garantizarse siempre.

Sin embargo, en España actualmente hay 300.000 niños en riesgo de perder el cuidado parental, de los cuales 48.000 ya se encuentran dentro del sistema de protección. Los niños, niñas y adolescentes en riesgo de perder el cuidado de sus familias y aquellos que ya lo han perdido se constituyen como nuestro grupo meta. Además de que su derecho a la familia ha sido violentado, ellos están más expuestos a sufrir explotación, abuso y negligencia durante su infancia y, al llegar a la edad adulta, de enfrentarse a mayores desafíos para adaptarse a la vida autónoma y participar activamente en la sociedad. Además, son más propensos a experimentar discriminación, exclusión social, inseguridad laboral, pobreza y problemas de salud.

Ellos son la razón de nuestra existencia y por los cuales desplegamos todos nuestros esfuerzos. Los niños sin cuidado parental deben ser una parte fundamental de la Agenda 2030, pues hay objetivos que no podrán alcanzarse si no son tenidos en cuenta.

Es posible hablar del impacto que ha tenido nuestro trabajo (y tendrá de futuro) en relación a cumplimiento de los ODS, en base a nuestro impacto en las vidas de los individuos y así estimar la medida en la cual contribuimos con las metas específicas de los ODS.

Dado nuestro impacto en las vidas de los exparticipantes, es razonable esperar que los resultados positivos que vemos en ellos igualmente deban verse reflejados en las vidas de los participantes actuales y futuros hasta 2030. Por ejemplo, en nuestros programas de acogimiento de tipo familiar y de fortalecimiento familiar apoyamos a los niños, niñas y jóvenes para que tengan acceso a una educación de calidad, reciban formación en habilidades desde el desarrollo de la primera infancia hasta la educación secundaria, formación profesional y en algunos casos hasta la educación universitaria. En promedio, en todas estas áreas relacionadas con educación y habilidades, el $82 \%$ de los exparticipantes han tenido resultados positivos. Dado que las metas de los ODS 4.2 y 4.5 se refieren específicamente a estas áreas de educación, establecemos que nuestro trabajo directamente contribuye a alcanzar estas metas. Podemos esperar, por tanto, que el $82 \%$ de nuestros participantes actuales y futuros sean positivamente impactados en términos de educación y habilidades, lo que equivaldría a 5,3 millones de niños y niñas hasta 2030. 
Esta misma lógica ha sido aplicada a las metas relacionadas con los ODS 1, 8, 10 y 16, según se ilustra en la figura a continuación:
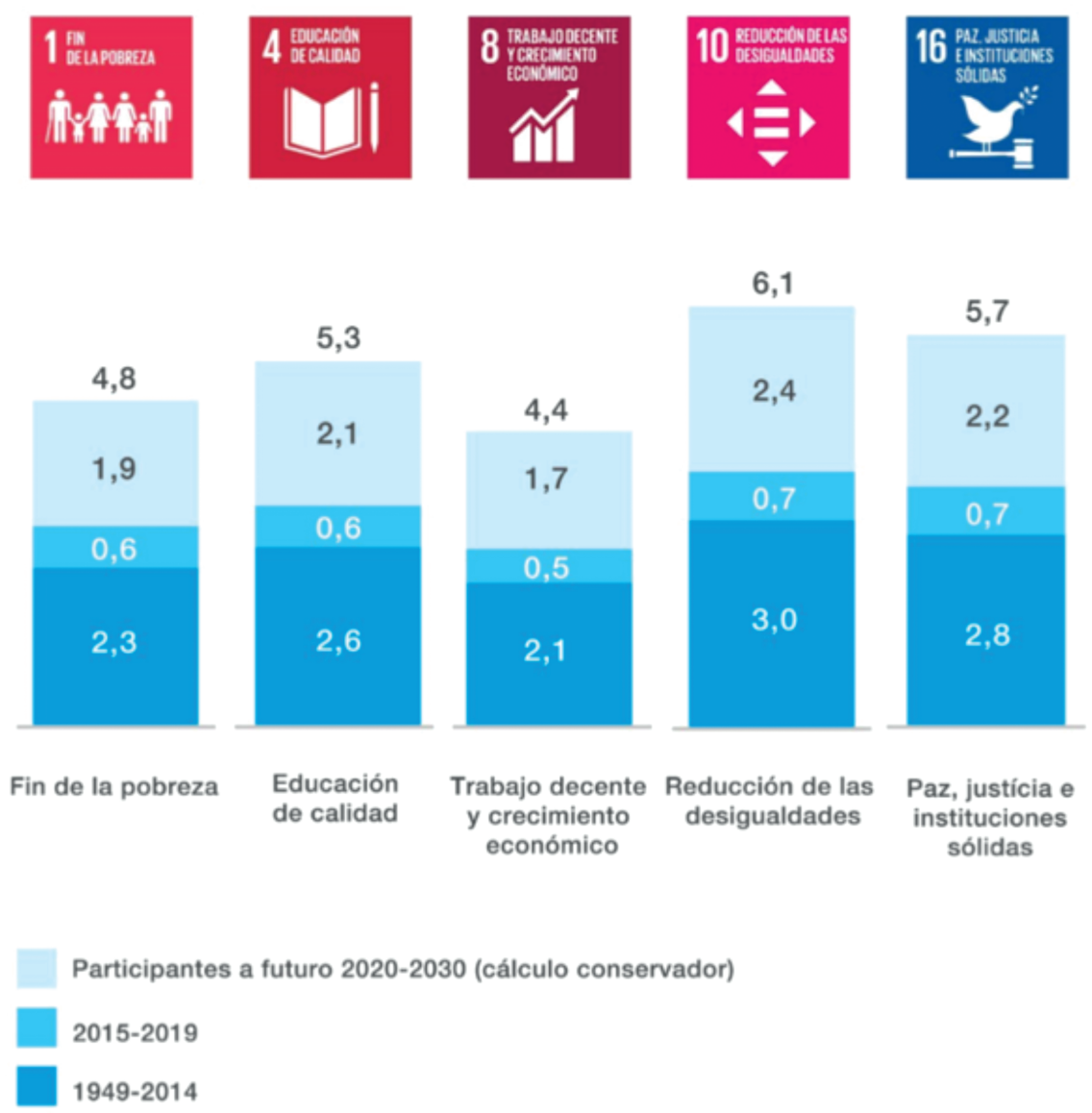

Además de los ODS anteriormente mencionados, también incidimos indirectamente sobre otros, como por ejemplo el ODS 3 que se refiere a 'salud y bienestar'. Los resultados de nuestro impacto han mostrado que el 93\% de los exparticipantes de nuestros programas gozan de buena salud y tienen acceso a servicios de atención médica pertinentes. En nuestros programas trabajamos junto con aliados locales para garantizar que niños y familias tengan un acceso adecuado a la atención médica. Por lo tanto, siguiendo el planteamiento antes expuesto, probablemente habremos impactado a seis millones de niños y niñas a través de nuestra contribución indirecta al ODS 3 hasta 2030. 
Para Aldeas, trabajar por y para los niños en situaciones de vulnerabilidad es nuestra razón de ser y existir. Cuando dialogamos con otras instituciones y particulares respecto a la importancia de prestar atención y cuidar de la infancia, el primer argumento en el que hacemos énfasis es que debemos HACERLO POR ELLOS. Por su presente. Porque el lugar en el que un niño o niña nace no debería determinar el acceso a sus derechos, condicionar su desarrollo y aprendizaje y limitar sus oportunidades futuras. Cuando esto sucede, como sociedad estamos fallando. Estamos quitándoles a estos niños la posibilidad de elegir qué hacer en su futuro. Les estamos quitando su libertad.

En una segunda instancia, es importante señalar que invertir en la infancia temprana trae consigo resultados positivos y rentables para la sociedad en su conjunto. Lo que suceda en esta etapa de la vida de las personas determina su futuro y son la base sobre la que se construyen las sociedades. De este modo, los países más prósperos son aquellos que han invertido más en la infancia (World Bank, 2011). Invertir en la educación, salud y en el apoyo y fortalecimiento estratégico de la familia en la primera infancia tiene como resultado la disminución de los índices de delincuencia, de los embarazos a edades tempranas y de inequidad; lo que contribuirá al incremento de la productividad y competitividad de las personas y, por lo tanto, impactará positivamente a la economía del país (Heckman, 2015).

Así, invertir esfuerzos en mejorar las condiciones que viven los niños y adolescentes implica no solo impactar en sus vidas y en los ODS directamente ligados a ellos, sino que tiene un efecto multiplicador. Trabajar con un niño, tiene impacto sobre su familia, sobre la institución a la que este asiste, sobre barrio en el que vive, y así sucesivamente. En definitiva, si un niño está bien, significa que su familia está bien y, si su familia está bien, es más fácil que la comunidad también lo esté. El potencial de escalabilidad que tiene invertir en infancia es innegablemente alto y positivo.

Cuidar a un niño es quizás la tarea más noble que tenemos los adultos, ya sea desde nuestra condición de padres, de educadores, de profesionales de la salud, o desde las propias responsabilidades de los dirigentes y líderes sociales y políticos. Una sociedad que se precie y sea sana, tiene que poder realizar todos los esfuerzos posibles para que ningún niño quede atrás. El principio de igualdad de oportunidades se genera desde la primera infancia y en este principio se va afianzando y construyendo la justicia social, que en definitiva es de lo que trata la Agenda 2030.

Si atendemos sus necesidades físicas, espirituales y afectivas, estaremos contribuyendo a que puedan llegar a ser aquello que desean, y este destino rara vez será incompatible con la creación de una sociedad más justa y mejor organizada. Debemos buscar juntos soluciones para conseguir que todos los niños y niñas vean realizados y respetados sus derechos, ya que es la mejor herramienta para la 
consecución de sociedades igualitarias, prosperas y justas; y la mejor fórmula posible para no dejar a nadie atrás.

Ahora, ¿cómo está España en relación al cumplimiento de los compromisos asumidos con la Agenda 2030? ¿Hablar de que estamos yendo hacia una sociedad más justa, igualitaria, próspera y respetuosa con el entono, es algo real? El reporte internacional SDG Index and Dashboards Report 2018 refleja cuál es la tendencia en el cumplimiento de los ODS en España hasta ese año. De acuerdo con los datos arrojados, deberíamos reforzar esfuerzos en casi la mitad de estos para cumplir los objetivos y mejorar la tendencia actual. Según la tendencia, los indicadores para los ODS 1, ODS 8, ODS 14, ODS 15 y ODS 16 permanecen estancados o están avanzando a un ritmo inferior al $50 \%$ de la tasa de crecimiento necesaria para alcanzar los ODS en 2030; mientras que para los ODS 10 y 17 los indicadores nos señalan que debemos hacer cambios profundos. Esta fotografía de España a 2018 nos permite analizar hacia dónde deben ir los esfuerzos del Estado, las empresas y las organizaciones del tercer sector. En el caso de Aldeas Infantiles SOS, nuestro trabajo incide precisamente en cinco de los siete ODS en los que existe un mayor riesgo de incumplimiento (ODS 1, ODS 8, ODS 10, ODS 16 y ODS17). Hoy reafirmamos, más que nunca, el compromiso asumido de responder de manera contundente al cumplimiento de estas metas.

Solo en la confluencia y suma de esfuerzos lograremos revertir las cifras que nos indican que, de seguir así, no llegaremos a la meta pactada para 2030; una meta que no tiene que ver con el mero cumplimiento de las cifras sino con las personas y con nuestra sociedad en su conjunto, siendo por tanto una llamada a la responsabilidad social de todos los actores que la conformamos.

La comunidad internacional tiene una oportunidad sin precedentes con los ODS. Si unimos fuerzas y todos los actores sociales ponemos algo de nuestra parte, el efecto será transformador. No podemos dejar pasar esta oportunidad, quizás la última, de trabajar juntos por un mundo mejor, para no dejar a nadie atrás, especialmente los niños que más nos necesitan. 



\title{
AUARA, EL COMPROMISO DE LA EMPRESA SOCIAL CON LOS ODS
}

\author{
Autor: Antonio Espinosa de los Monteros \\ hola@auara.org \\ CEO y cofundador de AUARA
}

\section{Resumen}

En este artículo AUARA, empresa social que comercializa agua mineral natural y destina el $100 \%$ de sus dividendos a llevar agua potable a países que no disponen de este recurso, vincula su actividad a los Objetivos de Desarrollo Sostenible (ODS) desde que inició su actividad, septiembre 2016, a la actualidad. La compañía ha logrado entrar en beneficios en 2018, reafirmando su modelo de impacto social.

Palabras clave: ODS; Agenda 2030; empresa social; sostenibilidad, agua.

\section{AUARA, a social enterprise commitment to SDG}

\author{
Abstract \\ In this article AUARA, a social company that sells natural mineral water \\ and allocates $100 \%$ of its dividends to bring drinking water to countries that do


not have this resource, links his activity to the Sustainable Development Goals (SDG) since it began its activity, September 2016, to the present. The company has achieved profits in 2018 , reaffirming its social impact model.

Keywords: SDG; Agenda 2030; social enterprise; sustainability; water.

Fecha de recepción: 18/11/2019.

Fecha de aceptación: 20/11/2019.

"Hemos llegado a un momento decisivo para la historia de la humanidad. Los pueblos del mundo nos han pedido que alumbremos el camino hacia un futuro prometedor y lleno de oportunidades. Y los estados miembros han respondido con la Agenda 2030 para el Desarrollo Sostenible”, con estas palabras Ban Ki-moon, entonces secretario general de Naciones Unidas, cargo que ocupó entre 2007-2017, anunciaba en la clausura la Asamblea General de la ONU, del 25 de septiembre de 2015, la aprobación de los 17 Objetivos de Desarrollo Sostenible (ODS) por parte de los estados miembros, que están marcando la agenda global para afrontar los desafíos globales que tenemos como Humanidad: lucha contra la pobreza y el hambre, disminución de la desigualdad, cambio climático -que ha derivado en emergencia climática-, fomento de la igualdad de género, de la educación o del acceso al agua limpia y saneamiento.

Hace cuatro años AUARA estaba en fase de concepción. Y hace cuatro años el mundo afrontaba los mismos problemas que en la actualidad, aunque parece que se han ido incrementando. El hambre afecta a 821 millones de personas y lleva tres años aumentando, según el El estado de la seguridad alimentaria y la nutrición en el mundo de la FAO (15 de julio de 2019). Más de 70 millones de personas $(70,8)$ se han visto obligadas a abandonar sus hogares por los conflictos armados o la persecución, según el Informe de ACNUR Tendencias Globales, desplazamiento forzado en 2018, que indica que 25,9 millones de personas son refugiadas y más de la mitad menores de edad. Además, en menos de dos años se ha pasado de hablar de cambio climático a emergencia climática y los científicos nos lo recuerdan. El IPCC, el Grupo Intergubernamental de Expertos en Cambio Climático, lanzaba una señal de alarma en su informe de 2018 para transitar hacia una economía descarbonizada si queríamos cumplir con el Acuerdo de París para frenar el cambio climático y limitar cuanto antes el calentamiento global a 1,5 grados, que podría alcanzarse entre 2030 y 2050 si las emisiones de CO2 continúan a este ritmo. 
En septiembre de 2016 nació AUARA. Justo en el primer aniversario de los ODS, pero cuando fundamos la empresa social -Pablo Urbano, director de Operaciones (CCO) y cofundador; Luis de Sande, director Financiero y cofundador, y yo como CEO y cofundador- no pensamos de inicio en cómo podíamos contribuir a la Agenda 2030, aunque ya lo estábamos haciendo, sin ser conscientes. Nuestro propósito era y es el de acabar con la mayor de las pobrezas, la falta de acceso a agua porque sin este recurso no hay posibilidad de desarrollo.

De este modo, nos constituimos como empresa social que destina el 100\% de sus dividendos a proyectos sociales para llevar agua potable a poblaciones que carecen de ella. Y para ello, tuvimos que mirar fuera de España porque todavía no existe esta figura jurídica y nos convertimos en la primera empresa social española certificada por el sello Social Enterprise Mark (Reino Unido) para garantizar en nuestro pacto de socios que no nos desviaríamos de nuestro compromiso fundacional.

AUARA significa en amhárico, idioma etíope, "tormenta de arena que se produce en desiertos como el de Afar, el lugar más cálido y seco del planeta”. Surgió con una clara y proactiva vocación social con la que invita a todo el mundo que la consume a convertir un acto cotidiano como beber agua, en un acto extraordinario. Y le pusimos ese nombre después de que me fuese como voluntario a Etiopía y participase en la construcción de un hospital junto a la ONG Amigos de Silva y me di cuenta que la causa principal de que la gente acudiera al hospital venía motivada por enfermedades vinculadas con la falta de acceso a agua potable.

Antes había participado como voluntario en Perú y Camboya, pero tras llegar de Etiopía me encontré con Pablo, que era amigo del colegio, y unimos el problema del agua con la empresa social y creamos AUARA. Yo soy arquitecto y Pablo ingeniero, pero necesitábamos a alguien que nos ayudara a poner en marcha el proyecto y apareció Luis.

Desde entonces no hemos parado de trabajar para cumplir con nuestro propósito, llevar agua a poblaciones que carecen de ella y facilitar su desarrollo. Recientemente, acabamos de demostrar que los proyectos de impacto social también son rentables: en nuestro tercer año de actividad (2018) hemos entrado en beneficios, alcanzado el equilibrio entre los ingresos y los costes. Era algo que no nos preocupaba, pero que traslada un mensaje a la sociedad de que la empresa social también puede ser rentable y contribuir a mejorar la vida de las personas.

Coincidiendo con el cuarto aniversario de los ODS, el pasado mes de septiembre, decidimos analizar nuestra contribución a los mismos. Y comprobamos, que además de impactar directamente en los referidos al ODS 1 Fin de la pobreza, al ODS 6 Agua Limpia y Saneamiento y al ODS 17 Alianzas, quien bebe AUARA está contribuyendo directamente con 15 de los 17 ODS. 
Compartimos, modestamente, la vinculación de nuestra actividad con la Agenda 2030 con la idea de que pueda servir de inspiración a otras empresas sociales, empresas u organizaciones para alinearse con los ODS.

ODS 1: Fin de la pobreza. La falta de acceso a agua potable es la pobreza más grande que existe porque sin agua no hay desarrollo posible. La ONU reconoció en 2010 que el acceso al agua potable y saneamiento son esenciales para la realización de todos los derechos humanos y desde AUARA tratamos de contribuir con los 50 proyectos de acceso a agua que hemos realizado en 15 países hasta la fecha.

ODS 2: Hambre cero. Si unimos que en el mundo hay 821 millones de personas que pasan hambre y que 3 de cada 10 personas carecen de acceso a servicios seguros de agua potable, según la $\mathrm{ONU}$, la lucha contra la pobreza y el hambre no se podrá cumplir sin que el agua entre en la ecuación del desarrollo sostenible.

El agua es el recurso natural que da sentido a la propiedad y al control de las tierras evitando entre otros problemas la emigración a ciudades que no hace en ocasiones más que trasladar de sitio el problema del hambre y la pobreza. La falta de agua en los cultivos es la principal causa de hambrunas en lugares en los que se practica una agricultura de subsistencia y cuando hay sequía tener acceso a agua para cultivar es un seguro de vida. En AUARA tratamos de fomentar la autosuficiencia alimentaria y la mejora de la nutrición con los proyectos que desarrollamos.

ODS 3: Salud y bienestar. El acceso a agua potable es el primer paso para asegurar la salud de las personas y contribuye a la disminución de las enfermedades generadas por el agua como cólera, fiebre tifoidea, meningitis, hepatitis o diarrea. Cada año, 3,5 millones de personas mueren en el mundo por no tener acceso a agua potable o por enfermedades relacionadas con el agua, según el World Water Council, que calcula que cerca de 1.000 niños fallecen cada día por las mismas causas. De este modo, la falta de agua se ha convertido en un arma devastadora que causa más mortalidad que la peor de las guerras.

A través de los 50 proyectos que hemos desarrollado en estos años hemos permitido que 28.411 personas tengan acceso a agua potable y saneamiento, lo que permite reducir hasta en un $80 \%$ las enfermedades con un impacto inmediato en la tasa de mortalidad infantil.

ODS 4: Educación de calidad. Cuando en una zona o poblado no hay acceso a agua, las madres y las niñas son las que tienen que recorrer varios kilómetros cada día para rellenar sus bidones en el río más cercano y llevarlos de vuelta a casa para el abastecimiento de la familia, lo que les impide asistir a la escuela y adquirir una formación que les permita mirar al futuro con esperanza. Por eso, tener acceso a agua permite que las niñas puedan estudiar.

ODS 5: Igualdad de género. Cuando se trata de ir a por agua, el género masculino no existe y las mujeres y las niñas son las responsables de ir a buscarla. Se estima que pueden emplear hasta 40.000 millones de horas en ir a por agua y la 
liberación de tiempo disponible ofrece un mayor tiempo de dedicación a los hijos, la posibilidad de ir a la escuela, la participación en nuevas actividades de la comunidad, la adquisición de habilidades sociales y, en definitiva, la promoción de la igualdad de derechos.

ODS 6: Agua limpia y saneamiento. De lo que nos podemos sentir más orgullosos en AUARA es de haber facilitado el acceso a agua potable y saneamiento a 28.411 personas desde nuestro lanzamiento en 2016. Somos ambiciosos y en 2020 queremos asegurar el agua potable a 50.000 personas, duplicando la cifra actual en poco más de un año.

Nuestros compromiso fundacional con el ODS 6 tuvo su recompensa, sin buscarla, al ser galardonados en la primera edición de los Reconocimientos go!ODS (octubre 2018), impulsados por la Red Española de Pacto Mundial y la Fundación Rafael del Pino.

ODS 7: Energía asequible y no contaminante. No impactamos directamente.

ODS 8: Trabajo decente y crecimiento económico. El agua es prosperidad. En el campo genera mayor rendimiento de los cultivos y su dotación a nivel de infraestructuras genera mejor calidad de vida en las viviendas, posibilidad de trabajo y desarrollo comunitario.

El impacto social de AUARA proviene por dos vías. Por una parte, la confianza de los consumidores en nuestra oferta -agua mineral, agua con gas y, próximamente, refrescos a través de Planet Drink- y de la colaboración con las empresas con las que llevamos a cabo los proyectos en terreno. Pero también se genera desde el equipo de AUARA, formado por más de 20 profesionales comprometidos con el impulso de una sociedad más próspera y no lo podíamos hacer de otro modo que desde la gestión responsable de personas que genera orgullo de pertenencia.

ODS 9: Industria, innovación e infraestructira. No impactamos directamente.

ODS 10: Reducción de las desigualdades. Como elemento básico para el desarrollo, el agua es el recurso desde el que se empieza a reducir las desigualdades. Desde nuestro lanzamiento, en septiembre de 2016, hemos puesto en marcha 50 proyectos en poblaciones en situación de pobreza extrema, pertenecientes a 15 países de África, Asia y América Central.

ODS 11: Ciudades y comunidades sostenibles. El acceso a un saneamiento seguro incide directamente en la salud y mejora de las condiciones de vida. Se estima que 6 de cada 10 personas, unos 4.500 millones de personas en todo el mundo, carecen de un saneamiento seguro, según la Organización Mundial de la Salud (OMS). Desde AUARA contribuimos a reducir esa cifra gracias a nuestros proyectos.

ODS 12: Producción y consumo responsables. La venta de botellas de agua es el vehículo que tenemos para poder llevar este recurso a personas que no la tienen. Cuando pusimos en marcha AUARA, después de analizar las diferentes opciones 
para comercializar agua, nos dimos cuenta que envasar el agua en material RPET $100 \%$ reciclado y $100 \%$ reciclable era la opción que menos impacto medioambiental tendría, convirtiéndonos en la primera empresa europea en apostar por envasar toda nuestra producción en este material. Desde entonces hemos reciclado más de 138 toneladas de plástico, que se han traducido en el ahorro de más de 226.000 litros de petróleo. Para 2020 pretendemos evitar el empleo de 10 millones de litros de petróleo en RPET y reutilizar el plástico de 90 millones de botellas.

ODS 13: Acción por el clima. Estamos comprometidos con minimizar el impacto ambiental de nuestro producto promoviendo envases más eficientes (RPET $100 \%$ reciclado y reciclable), botellas con un diseño que permite el transporte de un $20 \%$ más en cada palet o una flota comercial de vehículos híbridos. Apostamos por la economía circular para minimizar nuestro impacto medioambiental.

ODS 14: Vida submarina. Nos nutrimos de agua dulce y no salada, pero sabemos que la sostenibilidad de los océanos y recursos marinos es fundamental para nuestra supervivencia y el cuidado de la biodiversidad. Por eso, apostamos por no generar más plástico sino usar el que ya está para que no llegue al mar.

ODS 15: Vida de ecosistemas terrestres. La disponibilidad de agua promueve la sostenibilidad de las comunidades y su desarrollo, disminuye la degradación y desertificación de las tierras.

ODS 16: Paz, justicia e instituciones sólidas. Con motivo del Día Mundial del Agua en 2019, lanzamos una campaña de sensibilización con un mensaje impactante en sus botellas para simbolizar que el agua, o su ausencia, es un arma mortal que cada año acaba con la vida de 3,5 millones de personas, según el World Water Council. Así, la aportación de agua a las comunidades facilita a creación de instituciones eficaces, responsables e inclusivas.

ODS 17: Alianzas para lograr los objetivos. El 17 es el ODS estrella, sin él, el resto parecen incompletos. Sin darnos cuenta, hemos visto que este ODS da sentido a todo nuestro propósito, llevar agua a países que carecen de este recurso. Y lo hemos hecho posible gracias a la colaboración de las entidades no lucrativas y de las empresas, porque de otro modo no podríamos tener impacto social.

En la actualidad, AUARA colabora con 10 organizaciones para desarrollar sus proyectos: África Directo, Ayuda en Acción, Zerca y Lejos, Fundación Acción Geoda, Fundación Esperanza y Alegría, Fundación Kirira, Fundación Promoción Social, Sauce ONG, Ongawa y Manos Unidas. Cada uno de los 50 proyectos se han implementado de la misma manera, acompañando durante cinco años a las organizaciones españolas responsables de cada uno de ellos y que cuentan con un aliado de referencia sobre el terreno para garantizar su buen desarrollo.

Estos proyectos no se podrían llevar a cabo sin la confianza de las 75 empresas que confían en el agua de AUARA y a las que desarrollan proyectos propios como Calidad Pascual, Más Móvil, Ilunión, Ahorramás, Selecta, Room Mate, Veritas, 
Aveda, BBVA, Fundación Universitaria San Pablo-CEU, Coolook, El Ganso, Gaia Camp, Grupo Álava, Grupo Lalala, Grupo Larrumba e Isban.

Esta alianza ha permitido que generemos más de 21,6 millones de litros de agua en países en vías de desarrollo que han beneficiado a 28.411 personas.

Por cada litro de agua vendido, AUARA es capaz de generar cuatro litros de agua en países en vías de desarrollo mediante la construcción de pozos o la instalación de tanques de recogida de agua de lluvia. Sin duda, todos los que hacemos realidad AUARA nos sentimos orgullosos de mejorar la sociedad y de trabajar para seguir impulsando el impacto social. 